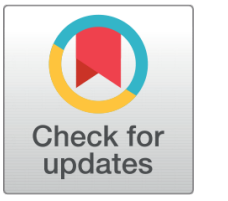

OPEN ACCESS

Received: 31.03 .2021

Accepted: 19.05.2021

Published: 05.06.2021

Citation: Nagalakshmi PSS, Vijaya N (2021) Numerical simulations of Darcy -Forchhiemer flow explored with carbon nanotubes under activation energy. Indian Journal of Science and Technology 14(20): 1661-1676. https://doi.org/ 10.17485/IJST/v14i20.544

* Corresponding author.

sathya.krishnat@gmail.com

Funding: None

Competing Interests: None

Copyright: (c) 2021 Nagalakshmi \& Vijaya. This is an open access article distributed under the terms of the Creative Commons Attribution License, which permits unrestricted use, distribution, and reproduction in any medium, provided the original author and source are credited.

Published By Indian Society for Education and Environment (iSee)

ISSN

Print: 0974-6846

Electronic: 0974-5645

\section{Numerical simulations of Darcy -Forchhiemer flow explored with carbon nanotubes under activation energy}

\author{
P S S Nagalakshmi ${ }^{1 *}$, N Vijaya ${ }^{1}$ \\ 1 Department of Mathematics, Koneru Lakshmaih Eduction Foundation, Deemed to be \\ University, Vaddeswaram, Guntur, 522502, Andhra Pradesh, India
}

\section{Abstract}

Objectives: To obtain the results of the computational analysis of threedimensional Darcy-Forchheimer flow concerning the hall current and Joule heating with Arrhenius activation energy explored using single-wall carbon nanotube and multi-wall carbon nanotube. Methods: The flow equations are modified to a nonlinear system of ordinary differential equations with adequate self-similarity functions. The solution to the modified system is evaluated by numerical techniques with python coding. Findings: In this study, the outcomes of engineering parameters are evaluated numerically. Influence of governing parameters on momentum, thermal, and concentration boundary layers are depicted graphically with different base fluids explored with single and multi-wall carbon nanotubes under Darcy -Forchheimer flow of nanofluid. Novelty: A favorable comparison is performed with previously available outcomes. The achieved results are almost like solutions obtained by other researchers. Carbon nanotubes are additionally used in an incredible range of applications in electronic, structural engineering, biomedical, energy management, and chemical processing.

Keywords: Activation energy; hall current with ion slip; joule heating; carbon nanotube; python

\section{Introduction}

Carbon Nanotubes are the only atomically thick tubes formed by a graphite layer enclosing hexagonal carbon atoms. The folding direction of the graphite foil is determined by the chiral vector $\mathrm{Ch}(\mathrm{n}, \mathrm{m})$, so CNTs can be divided into chiral nanotubes $(n, m)$, zigzag nanotubes $(n, 0)$, and chair nanotubes $(n, n)$. Carbon nanotubes have become one of the most studied materials in the past decade due to their promising mechanical, electrical, and thermal properties. Single wall nanotubes (SWCNT) contain a cylinder, while multilayer nanotubes (MWCNT) have multiple concentric cylinders separated by almost equal distances because van der Wall's graphite plates are stacked on top of each other.

Nanofluid is an innovative type of resolution, which is provided by dispersing nanoscale materials (nanoparticles, nanofibers, nanotubes, nanowires, nanorods, nanofilters or droplets) in a base fluid. Creep associated with nanofluids is used 
in biomedicine, that is, in radiotherapy of cancer. Choi demonstrated in 1995 that an advanced heat transfer recovery technology uses nano-scale particles in the base fluid. The literature on this subject is very rich. Effect of Joule Heating and Thermal Radiation in Flow of Third Grade Fluid over Radiative Surface obtained by Hayat et al. ${ }^{(1)}$. Similarity solution to three-dimensional boundary layer flow of second grade nanofluid past a stretching surface with thermal radiation and heat source/sink solved by Hayat et al. ${ }^{(2)}$. Sreenivasulu and Reddy ${ }^{(3)}$ presented the lie group analysis for boundary layer flow of nanofluids near the stagnation-point over a permeable stretching surface embedded in a porous medium in the presence of radiation and heat generation/absorption. Influence of viscous dissipation and Joule heating on MHD bio-convection flow over a porous wedge in the presence of nanoparticles and gyrotactic microorganisms has been explored by Khan et al. ${ }^{(4)}$. Afshar et $\mathrm{al}^{(5)}$ considered the effect of dispersed nanoparticles on thermophysical properties of nanofluid and heat transfer coefficients. Influence of joule heating and non-linear radiation on MHD 3D dissipating Flow of casson nanofluid past a non-linear stretching sheet has been analyzed by Sreenivasulu et al. ${ }^{(6)}$. Shahzada et al. ${ }^{(7)}$ studied the numerical simulation of magnetohydrodynamic Jeffrey nanofluid flow and heat transfer over a stretching sheet considering joule heating and viscous dissipation. Simultaneous impacts of joule heating and variable heat source/sink on MHD 3D flow of carreau-nano liquids with temperature dependent viscosity has been solved by Ramana Reddy et al. ${ }^{(8)}$. Gholinia et al. ${ }^{(9)}$ obtained the results of investigation on ethylene glycol nanofluid flow over a vertical permeable circular cylinder under effect of magnetic field. Jodh Singh et al. ${ }^{(10)}$ described the heat transfer using nanofluid. Effect of Joule heating on the flow over an exponentially stretching sheet with convective thermal condition has been conferred by Srinivasacharya et al. ${ }^{(11)}$. Magneto hydrodynamicAA7072AA7075/methanol hybrid nanofluid flow above an uneven thickness surface with slip effect has been propounded by Iskanderet al. ${ }^{(12)}$. In 1856, Henry Darcy forced the pioneers to add a homogeneous liquid to the flow through the porous medium. However, the classic Darcy's law does not apply to flows with huge velocity and high permeability. To overcome this shortcoming, Forchheimer subsequently modified the Darcian velocity expression by adding the square of the velocity to the moment equation to estimate the collision velocity in the moment equation, and to evaluate the influence of inertia and the flux of physical phenomena. The term "Forchheimer" was first coined by Muscat and is usually used for huge Reynolds numbers, because the filtration rate is actually very high and it creates secondary resistance to the porous medium in the bending moment equation. Importance of Darcy-Forchheimer porous medium in 3D convective flow of carbon nanotubes shown by Alzahrani ${ }^{(13)}$. Numerical study of Darcy-Forchheimer model with activation energy subject to chemically reactive species and momentum slip of order two analyzed by Majeed et.al ${ }^{(14)}$. A revised model for Darcy-Forchheimer three-dimensional flow of nanofluid subject to convective boundary condition explained by Muhammad et.al ${ }^{(15)}$. Darcy-Forchheimer flow of hydromagnetic nanofluid over a stretching/shrinking sheet in a thermally stratified porous medium with second order slip, viscous and Ohmic dissipations effects results obtained by Vishnu Ganesh et.al ${ }^{(16)}$. Hosseinzadeh et.al ${ }^{(17)}$ investigated on crossfluid flow containing motile gyrotactic microorganisms and nanoparticles over a three-dimensional cylinder. Hosseinzadeh et.al ${ }^{(18)}$ studied on entropy generation of three-dimensional Bödewadt flow of water and hexanol base fluid suspended by $\mathrm{Fe}_{3} \mathrm{O}_{4}$ and $\mathrm{MoS}_{2}$ hybrid nanoparticles.

Investigators scrutinized the onset of binary chemical reaction with an activation energy, joule heating and hall current with ion slip in Darcy-Forchheimer flow of carbon nanotubes over a variable thickness of stretched surface through python. Python with the BVP has been established for the solutions of ODEs. The graphical portrayal is depicted and tables are structured with elaborated. Researchers found that this is the first study using python code as compared to related literature.

\section{Materials and Methods}

We scrutinize steady 3D magnetohydrodynamic (MHD) convective Darcy -Forchheimer flow explored with carbon nanotubes over a variable thickness of stretched under the influence of Joule heating, hall current with ion slip, and Arrhenius activation energy. Here the fluid is electrically conducting in the presence of applied magnetics $\vec{B}=\left(\mathbf{0}, \mathbf{0}, B_{\mathbf{0}}\right)$ and electric $\vec{E}=\left(\mathbf{0},-E_{\mathbf{0}}, \mathbf{0}\right)$ field. The flow is induced by a stretching Surface in two nearby $x$ and y directions with velocity $p$, q respectively. The electric and magnetic fields were carried out through Ohm's law. The induced magnetic flux and Hall current effects are ignored subject to small magnetic Reynolds numbers. Both the electrical and magnetic fields contribute to the momentum and thermal boundary layer equations. Furthermore, the concentration equation deals with the impact of Arrhenius energy of activation with binary reaction. In this clarification, the Brownian and thermophoresis nanoparticles are also engaged. The relevant equations in the afforested conditions can be expressed as follows (14):

$$
\frac{\partial p}{\partial x}+\frac{\partial q}{\partial y}+\frac{\partial r}{\partial z}=0
$$




$$
\begin{gathered}
\left(p \frac{\partial p}{\partial x}+q \frac{\partial p}{\partial y}+r \frac{\partial p}{\partial z}-2 \Omega q\right) \rho_{\mathrm{nf}}=\mu_{\mathrm{nf}} \frac{\partial^{2} p}{\partial z^{2}}-\sigma_{\mathrm{nf}} B_{0}^{2} \frac{(q \mathrm{me}-p \mathrm{ne})}{\mathrm{me}^{2}+\mathrm{ne}^{2}}-\frac{\mu_{\mathrm{nf}}}{k^{*}} p-\frac{c_{f} \rho_{\mathrm{nf}}}{\sqrt{k^{*}}} p^{2}+\sigma_{\mathrm{nf}} E_{0} B_{0} \\
\left(p \frac{\partial q}{\partial x}+q \frac{\partial q}{\partial y}+r \frac{\partial q}{\partial z}-2 \Omega p\right) \rho_{\mathrm{nf}}=\mu_{\mathrm{nf}} \frac{\partial^{2} q}{\partial z^{2}}-\sigma_{\mathrm{nf}} B_{0}^{2} \frac{(q \mathrm{me}-p \mathrm{ne})}{\mathrm{me}^{2}+\mathrm{ne}^{2}}-\frac{\mu_{\mathrm{nf}}}{k^{*}} q-\frac{c_{f} \rho_{\mathrm{nf}}}{\sqrt{k^{*}}} q^{2}+\sigma_{\mathrm{nf}} E_{0} B_{0} \\
\left(p \frac{\partial T}{\partial x}+q \frac{\partial T}{\partial y}+r \frac{\partial T}{\partial z}\right)\left(\rho c_{p}\right)_{\mathrm{nf}}=K_{\mathrm{nf}} \frac{\partial^{2} T}{\partial z^{2}}-\frac{\partial q_{r}}{\partial z}+\mu_{\mathrm{nf}} \frac{\partial p}{\partial z}+\sigma_{\mathrm{nf}}\left(\frac{(p+q)}{\mathrm{me}^{2}+\mathrm{ne}^{2}} B_{0}-E_{0}\right)^{2} \\
\left(p \frac{\partial C}{\partial x}+q \frac{\partial C}{\partial y}+r \frac{\partial C}{\partial z}\right)=D_{B} \frac{\partial^{2} C}{\partial z^{2}}-k_{r}^{2}\left(C-C_{\infty}\right)\left(\frac{T}{T_{\infty}}\right)^{n} \exp \left(\frac{-E^{*}}{\mathrm{kT}}\right)+\frac{D_{T}}{T_{\infty}} \frac{\partial^{2} T}{\partial z^{2}}
\end{gathered}
$$

Subject to the boundary conditions at $z \rightarrow 0$

$$
\begin{array}{r}
p=p_{1}\left(\exp \left(\frac{x+y+c}{L}\right)\right)^{m}, q=q_{1}\left(\exp \left(\frac{x+y+c}{L}\right)\right)^{m}, r=0 \\
-K_{\mathrm{nf}} \frac{\partial T}{\partial z}=-h_{f}\left(T_{w}-T\right), \frac{\partial C}{\partial z}=-h_{m}\left(C_{w}-C\right)
\end{array}
$$

Subject to the boundary conditions at $z \rightarrow \infty$

$$
p=q=0, T=T_{\infty}, C=C_{\infty}
$$

Here $\mathrm{p}, \mathrm{q}$, and $\mathrm{r}$ are the velocity components in $\mathrm{x}, \mathrm{y}$, and $\mathrm{z}$ directions respectively $\mu_{n f}$ dynamic nanofluid viscosity, $\mathrm{m}_{e}=1+\mathrm{n}_{i} \mathrm{n}_{e}$, where $\mathrm{n}_{i}$ is denoted the ion-slip while $\mathrm{n}_{e}$ is the hall current parameters, $\rho_{n f}$ density of nanofluid, $\mathrm{K}_{n f}$ thermal conductivity of nanofluid, $\sigma_{n f}$ electrical conductivity of nanofluid , $\mathrm{c}_{f}$ inertia resistance force, $\mathrm{k}^{*}$ permeability of porous medium, $\mathrm{c}_{p}$ specific heat $, \mathrm{D}_{B}, \mathrm{D}_{T}$ Brownian and thermal diffusion coefficient, $\mathrm{h}_{f}, \mathrm{~h}_{m}$ the wall heat and wall mass transport coefficients. Moreover, $\mathrm{n}$ is the fitted rate constant $(-1<\mathrm{n}<1), \mathrm{k}_{r}$ the reaction rate, $\mathrm{E}^{*}$ the activation energy, $\mathrm{k}$ the Boltzmann constant, whole expression $k_{r}^{2}\left(\frac{T}{T_{\infty}}\right)^{n} \exp \left(\frac{-E^{*}}{\mathrm{kT}}\right)$ entitled as modified Arrhenius equation. For thermal radiation we utilize the Rossel estimation, and then the radiative heat flux is defined as

$$
q_{r}=\frac{-16 \sigma^{*} T_{\infty}^{3}}{k^{s}} \frac{\partial T}{\partial z}
$$

Here $\sigma^{*}, \mathrm{k}^{\mathrm{s}}$ are the Stefan-Boltzmann constant and mean absorption coefficient, respectively.

Nanofluid parameters:

$$
\begin{gathered}
v_{\mathrm{nf}}=\frac{\mu_{\mathrm{nf}}}{\rho_{\mathrm{nf}}}, \mu_{\mathrm{nf}}=\frac{\mu_{\mathrm{bf}}}{(1-\varphi)^{2.5}}, \rho_{\mathrm{nf}}=(1-\varphi) \rho_{\mathrm{bf}}+\varphi \rho_{\mathrm{CNT}},\left(\rho c_{p}\right)_{\mathrm{nf}}=(1-\varphi)\left(\rho c_{p}\right)_{\mathrm{bf}}+\varphi\left(\rho c_{p}\right)_{\mathrm{CNT}} \\
\frac{K_{\mathrm{nf}}}{K_{\mathrm{bf}}}=\frac{(1-\varphi)\left(K_{\mathrm{CNT}}-K_{\mathrm{bf}}\right)+2 \varphi K_{\mathrm{CNT}} \ln \left(\frac{K_{\mathrm{CNT}}+K_{\mathrm{bf}}}{2 K_{\mathrm{bf}}}\right)}{(1-\varphi)\left(K_{\mathrm{CNT}}-K_{\mathrm{bf}}\right)+2 \varphi K_{\mathrm{bf}} \ln \left(\frac{K_{\mathrm{CNT}}+K_{\mathrm{bf}}}{2 K_{\mathrm{bf}}}\right)}, \frac{\sigma_{\mathrm{nf}}}{\sigma_{\mathrm{bf}}}=1+\frac{3\left(\sigma_{\mathrm{CNT}}-\sigma_{\mathrm{bf}}\right) \varphi}{\left(\sigma_{\mathrm{CNT}}+2 \sigma_{\mathrm{bf}}\right)-\left(\sigma_{\mathrm{CNT}}-\sigma_{\mathrm{bf}}\right) \varphi}
\end{gathered}
$$

Suitable conversions:

Let us consider

$$
\begin{gathered}
p=P f^{\prime}(\eta), q=P g^{\prime}(\eta), r=-\left(\frac{m v_{\mathrm{bf}} P}{2 L}\right)^{0.5}\left((f+g)+\eta\left(f^{\prime}+g^{\prime}\right)\right) \\
\theta(\eta)=\frac{T-T_{\infty}}{T_{w}-T_{\infty}}, h(\eta)=\frac{c-C_{\infty}}{C_{w}-C_{\infty}}, \quad P=p_{1}\left(\exp \left(\frac{x+y+c}{L}\right)\right)^{m}, \eta=\left(\frac{\mathrm{mP}}{2 v_{\mathrm{bf}} L}\right)^{0.5} z
\end{gathered}
$$


Here $\mathrm{p}_{1}, \mathrm{c}$, and $\mathrm{m}$ are constants and $\mathrm{L}$ is the reference distance.

The above transformations satisfy Eq. (1) automatically and Eqs. (2)- (5) with Eqs. (6) and (7) reduce to the following ODEs

$$
\begin{aligned}
& A_{2} f^{\prime \prime \prime}-K_{1}(M 1) A_{5} \frac{\left(\mathrm{meg}^{\prime}-\mathrm{ne} f^{\prime}\right)}{\mathrm{me}^{2}+\mathrm{ne}^{2}}-\frac{A_{2} K_{1}}{\mathrm{P}_{0}} f^{\prime}-(F i) K_{1} A_{1}\left(f^{\prime}\right)^{2} \\
& +(M 1)(E 1) K_{1} A_{5}-A_{1}\left(2\left(f^{\prime}\right)^{2}+2 g^{\prime} f^{\prime}-f^{\prime \prime} f-f^{\prime \prime} g-2 \varepsilon g^{\prime}\right)=0 \\
& A_{2} g^{\prime \prime \prime}-K_{1}(M 1) A_{5} \frac{\mathrm{meg}^{\prime}-\mathrm{nef}^{\prime}}{\mathrm{me}^{2}+\mathrm{ne}^{2}}-\frac{A_{2} K_{1}}{\text { Po }} g^{\prime}-(F i) K_{1} A_{1}\left(g^{\prime}\right)^{2} \\
& +(M 1)(E 1) K_{1} A_{5}-A_{1}\left(2\left(g^{\prime}\right)^{2}+2 g^{\prime} f^{\prime}-g^{\prime \prime} g-g^{\prime \prime} f-2 \varepsilon f^{\prime}\right)=0 \\
& \begin{array}{c}
A_{4}\left(\left(1+\frac{4}{3} \frac{R}{A_{4}}\right) \theta^{\prime \prime}+\frac{(M 1) K_{1}(\operatorname{Pr})(\mathrm{Ec}) A_{5}}{A_{4}}\left(\frac{f^{\prime}+g^{\prime}}{m \mathrm{e}^{2}+\mathrm{ne}^{2}}-(E 1)\right)^{2}+\frac{A_{1}}{A_{4}}(\mathrm{Pr})(\mathrm{Ec})\left(f^{\prime \prime}\right)^{2}\right) \\
-(\operatorname{Pr}) A_{3}\left(2 f^{\prime} \theta+2 g^{\prime} \theta-\theta^{\prime} f-\theta^{\prime} g\right)=0
\end{array} \\
& \left(h^{\prime \prime}+\frac{\mathrm{Nt}}{\mathrm{Nb}} \theta^{\prime \prime}\right)-K_{1}(\operatorname{Pr})(\mathrm{Le})(\delta 2) h(1+(\delta 1) \theta)^{m} \exp \left(\frac{-E}{1+\delta_{1} \theta}\right) \\
& -S c\left(2 h f^{\prime}+2 h g^{\prime}-f h^{\prime}-g h^{\prime}\right)=0 \\
& \begin{array}{c}
f(0)=0, g(0)=0, f^{\prime}(0)=1, g^{\prime}(0)=\beta \\
\theta^{\prime}(0)=-\left(\frac{2}{m}\right)^{0.5}\left(\frac{1}{A_{4}}\right) \gamma_{T}(1-\theta(0)), h^{\prime}(0)=-\left(\frac{2}{m}\right)^{0.5} \gamma_{C}(1-h(0)), \\
f^{\prime}(\infty)=0, g^{\prime}(\infty)=0, \theta(\infty)=0, h(\infty)=0
\end{array}
\end{aligned}
$$

Dimensionless parameters:

$$
\begin{gathered}
A_{1}=(1-\varphi)+\varphi \frac{\rho_{\mathrm{CNT}}}{\rho_{\mathrm{bf}}}, A_{2}=\frac{1}{(1-\varphi)^{2.5}}, A_{3}=(1-\varphi)+\varphi \frac{\left(\rho c_{p}\right)_{\mathrm{CNT}}}{\left(\rho c_{p}\right)_{\mathrm{bf}}} \\
A_{4}=\frac{(1-\varphi)\left(K_{\mathrm{CNT}}-K_{\mathrm{bf}}\right)+2 \varphi K_{\mathrm{CNT}} \ln \left(\frac{K_{\mathrm{CNT}}+K_{\mathrm{bf}}}{2 K_{\mathrm{bf}}}\right)}{(1-\varphi)\left(K_{\mathrm{CNT}}-K_{\mathrm{bf}}\right)+2 \varphi K_{\mathrm{bf}} \ln \left(\frac{K_{\mathrm{CNT}}+K_{\mathrm{bf}}}{2 K_{\mathrm{bf}}}\right)}, A_{5}=1+\frac{3\left(\sigma_{\mathrm{CNT}}-\sigma_{\mathrm{bf}}\right) \varphi}{\left(\sigma_{\mathrm{CNT}}+2 \sigma_{\mathrm{bf}}\right)-\left(\sigma_{\mathrm{CNT}}-\sigma_{\mathrm{bf}}\right) \varphi} \\
K_{1}=\frac{1}{m}, \beta=\frac{q_{1}}{p_{1}}, M 1=\frac{2 \sigma_{\mathrm{bf}} B_{0}^{2} L}{\rho_{\mathrm{bf}} P}, E 1=\frac{E_{0}}{\mathrm{~PB}{ }_{0}}, \mathrm{Po}=\frac{\mathrm{Pk}^{*}}{2 v_{\mathrm{bf}} L}, F i=\frac{2 c_{f} L}{\sqrt{k^{*}}}, R=\frac{4 \sigma^{*} T_{\infty}^{3}}{k^{s} k_{\mathrm{bf}}}, \varepsilon=\frac{2 L \Omega}{P} \\
\gamma_{T}=\frac{h_{f}}{K_{\mathrm{bf}}}\left(\frac{v_{\mathrm{bf}} L}{P}\right)^{0.5}, \gamma_{C}=\frac{h_{f}}{D_{B}}\left(\frac{v_{\mathrm{bf}} L}{P}\right)^{0.5}, \operatorname{Pr}=\frac{\left(\rho c_{p}\right)_{\mathrm{bf}} v_{\mathrm{bf}}}{k}, E c=\frac{P^{2}}{\left(c_{p}\right)_{\mathrm{bf}} \Delta T}, \mathrm{Sc}=\frac{v_{\mathrm{bf}}}{D_{B}} \\
N t=\frac{\left(\rho c_{p}\right)_{\mathrm{CNT}} D_{T} \Delta T}{v_{\mathrm{bf}} T_{\infty}\left(\rho c_{p}\right)_{\mathrm{bf}}}, \mathrm{Nb}=\frac{\left(\rho c_{p}\right)_{\mathrm{CNT}} D_{B} \Delta C}{v_{\mathrm{bf}}\left(\rho c_{p}\right)_{\mathrm{bf}}}, E=\frac{E^{*}}{\mathrm{kT}_{\infty}}, \delta 1=\frac{\Delta T}{T_{\infty}}, \delta 2=\frac{2 k_{r}^{2} L}{P} .
\end{gathered}
$$

Engineering parameters

Engineering parameters like skin friction coefficient $\mathrm{C}_{f x}, \mathrm{C}_{f y}$, local Nussult $\mathrm{Nu}_{x}$, and Sherwood $\mathrm{Sh}_{x}$ numbers are defined as

$$
C_{\mathrm{fx}}=\frac{\tau_{\mathrm{wx}}}{\rho_{\mathrm{bf}} P^{2}}, C_{\mathrm{fy}}=\frac{\tau_{\mathrm{wy}}}{\rho_{\mathrm{bf}} P^{2}}, \mathrm{Nu}_{x}=\frac{\mathrm{xq}_{w}}{K_{\mathrm{bf}} \Delta T}, \mathrm{Sh}_{x}=\frac{\mathrm{xJ}_{w}}{D_{B} \Delta C}
$$


in which $\tau_{w}, \mathrm{q}_{w}$, and $\mathrm{J}_{w}$ denote the surface shear stress, the surface heat flux and the surface mass flux respectively. Here

$$
\tau_{w_{x}}=\mu_{\mathrm{nf}}\left(\frac{\partial p}{\partial z}\right)_{z=0}, \tau_{\mathrm{wy}}=\mu_{\mathrm{nf}}\left(\frac{\partial q}{\partial z}\right)_{z=0}, q_{w}=-K_{\mathrm{nf}}\left(\frac{\partial T}{\partial z}\right)_{z=0}, J_{w}=-D_{B}\left(\frac{\partial C}{\partial z}\right)_{z=0} .
$$

Therefore, the dimensionless forms of overhead quantities are

$$
\begin{aligned}
& \left(\mathrm{Re}_{x}\right)^{0.5} C_{\mathrm{fx}}=\left(\frac{m}{2}\right)^{0.5} \frac{1}{(1-\varphi)^{2.5}} f^{\prime \prime}(0),\left(\mathrm{Re}_{x}\right)^{0.5} C_{\mathrm{fy}}=\left(\frac{m}{2}\right)^{0.5} \frac{1}{(1-\varphi)^{2.5}} g^{\prime \prime}(0), \\
& \left(\mathrm{Re}_{x}\right)^{0.5} \mathrm{Nu}_{x}=-\left(\frac{m}{2}\right)^{0.5}\left(\frac{(1-\varphi)\left(K_{\mathrm{CNT}}-K_{\mathrm{bf}}\right)+2 \varphi K_{\mathrm{CNT}} \ln \left(\frac{K_{\mathrm{CNT}}+K_{\mathrm{bf}}}{2 K_{\mathrm{bf}}}\right)}{(1-\varphi)\left(K_{\mathrm{CNT}}-K_{\mathrm{bf}}\right)+2 \varphi K_{\mathrm{bf}} \ln \left(\frac{K_{\mathrm{CNT}}+K_{\mathrm{bf}}}{2 K_{\mathrm{bf}}}\right)}\right) \theta^{\prime}(0), \\
& \left(\operatorname{Re}_{x}\right)^{0.5} \mathrm{Sh}_{x}=-\left(\frac{m}{2}\right)^{0.5} h^{\prime}(0),
\end{aligned}
$$

in which $\mathrm{Re}_{x}=\frac{\mathrm{xP}}{v_{\mathrm{bf}}}$ is the local Reynolds number.

\section{Results and discussion}

To attain this, the BVP scheme is engaged to discretize the governing equations into a well-ordered problem. For this determination, researchers revise equations (11)-(15) into first-order differential systems. Thus, in the present study, the value of $\eta=\eta$ max is taken to be 10 , and step-size is taken to be $\Delta \eta=0.01$ with a relative error tolerance of $10-5$. The relative study of present results with those obtained by Hayat et.al ${ }^{(1)}$ is shown in Tables 1,2 and 3. When compared to the other method obtained by Hayat et.al ${ }^{(1)}$ while equating the parameters of momentum, thermal, and concentration to zero for getting accuracy through python code.

Table 1. Accuracy assessment of $\mathrm{f}^{11}(0)$, when $\mathrm{m}=2$, and remaining parameters of momentum-thermo-physical and concentration are equated to zero

\begin{tabular}{lll}
\hline$B$ & Hayat et.al $^{(2)}$ & Present \\
\hline 0.0 & 1.28180856 & 1.281831634409684 \\
0.5 & 1.56988846 & 1.569704938336608 \\
1.0 & 1.81275105 & 1.812759680612502 \\
\hline
\end{tabular}

Table 2. Accuracy assessment of $\mathrm{g}^{11}(0)$, when $\mathrm{m}=2$, and remaining parameters of momentum-thermo-physical and concentration are equated to zero.

\begin{tabular}{lll}
\hline$B$ & Hayat et.al $^{(2)}$ & Present \\
\hline 0.0 & 0 & 0 \\
0.5 & 0.78494423 & 0.7848524691689054 \\
1.0 & 1.81275105 & 1.8127596806113822 \\
\hline
\end{tabular}

Table 3. Accuracy assessment of $\theta^{1}(0)$, when $\mathrm{m}=2, \operatorname{Pr}=0.7$ and remaining parameters of momentum-thermo-physical and concentration are equated to zero.

\begin{tabular}{lll}
\hline$B$ & Hayat et.al & Present \\
\hline 0.0 & -0.42589 & -0.44050 \\
0.25 & -0.47616 & -0.485435 \\
0.50 & -0.52161 & -0.526539 \\
0.75 & -0.56339 & -0.564776 \\
1.0 & -0.60228 & -0.599231 \\
\hline
\end{tabular}


The framework of this study is to scrutinize the properties of Arrhenius activation energy, Joule heating, and hall current with ion-slip with binary chemical reaction on steady 3D magnetohydrodynamic (MHD) convective Darcy -Forchheimer flow explored with carbon nanotubes over a variable thickness of the stretched sheet. Python with a BVP scheme has been executed to elucidate the present ODEs. The boundary layers of numerous parameters on momentum, thermal, and concentration is portrayed and discussed.

\section{Execution of Electric parameter(E1) on $\mathbf{f}^{1}(\eta), \mathbf{g}^{1}(\eta), \theta(\eta)$, and $\mathbf{h}(\eta)$}

To disclose the enactment of the electric parameter(E1) on momentum, thermal and concentration boundary layers are exposed from Figures 1, 2, 3 and 4. Physically, increasing the values of electric parameter boost the momentum boundary layer, thermal boundary layer, but in contrast, results appear in the concentration boundary layer. This causes that higher estimation of electric parameter relates to intensity of electric and magnetic field.

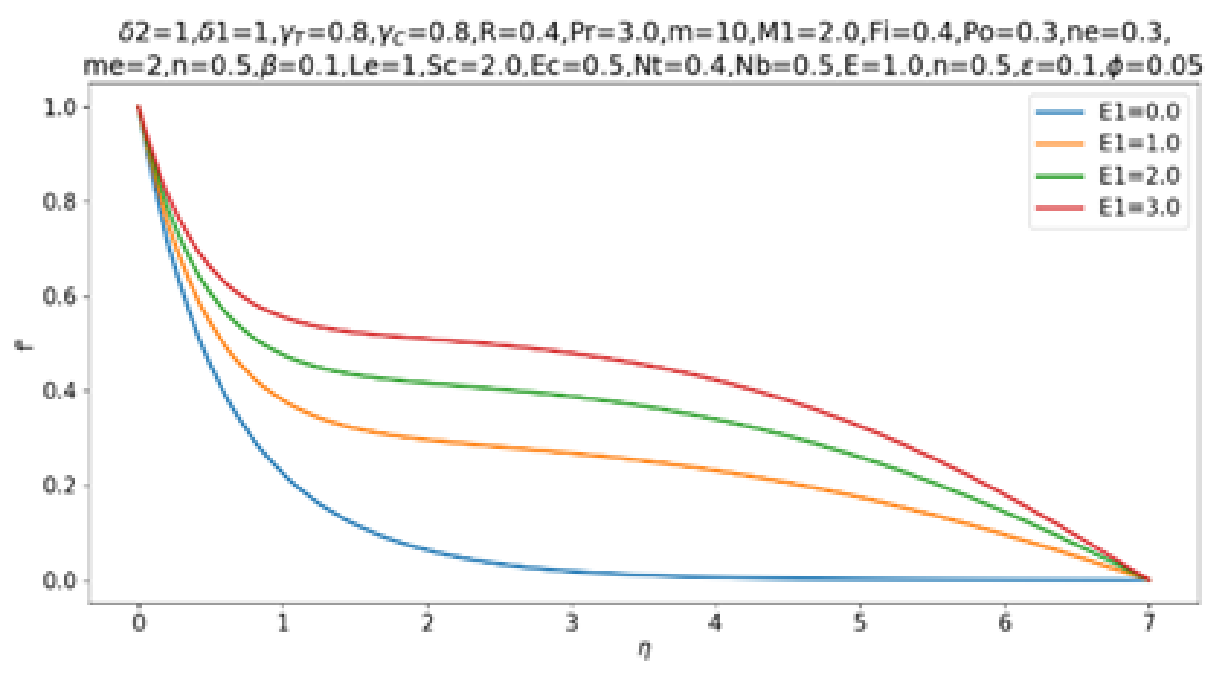

Fig 1. Horizontal velocity $\left[\mathrm{f}^{1}(\eta)\right]$ via Electric parameter (E1)

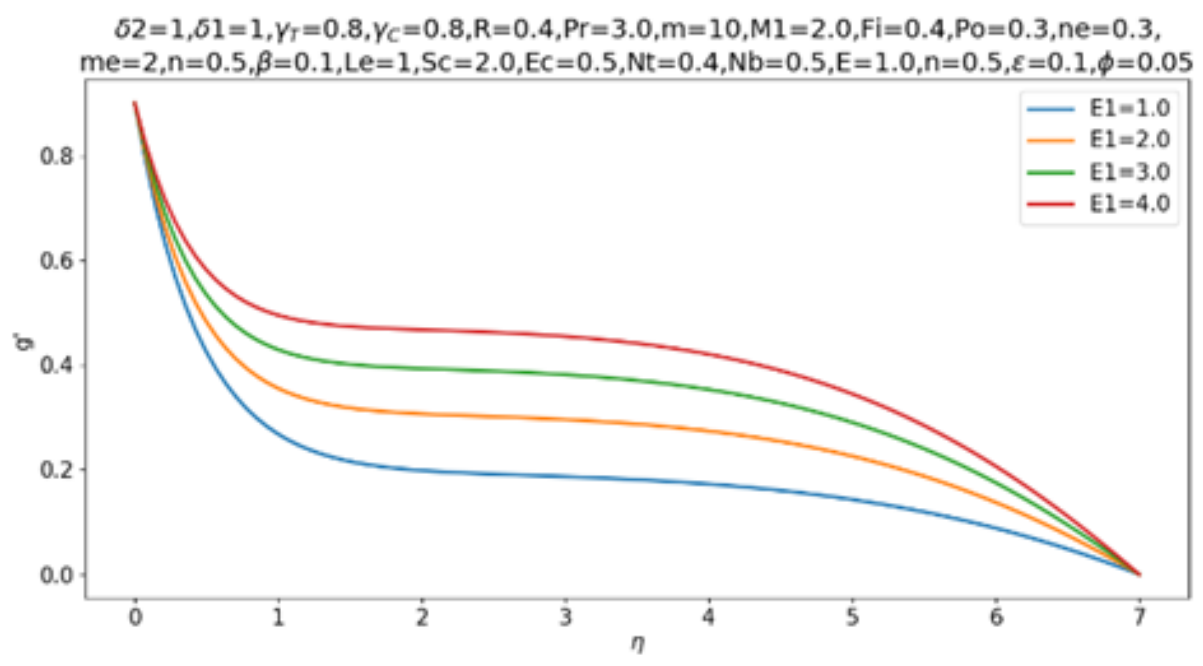

Fig 2. Vertical velocity $\left[g^{1}(\eta)\right]$ via Electric parameter (E1) 


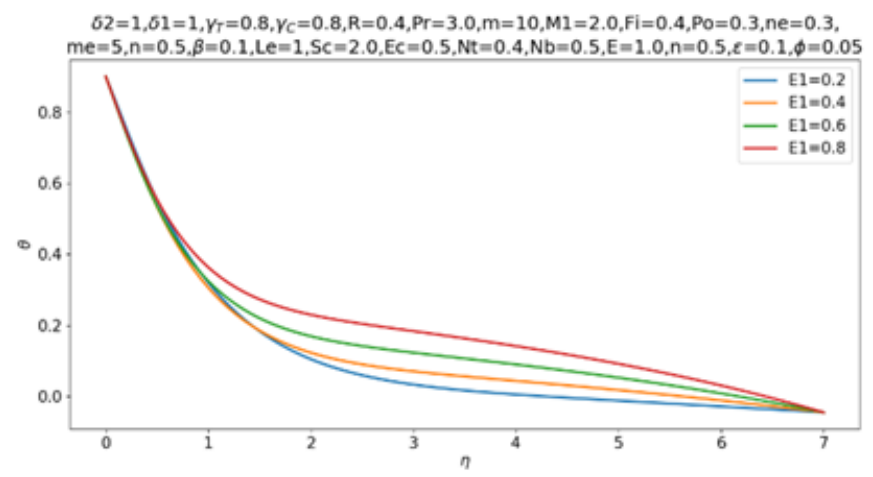

Fig 3. Temperature $[\theta(\eta)]$ via Electric parameter (E1)

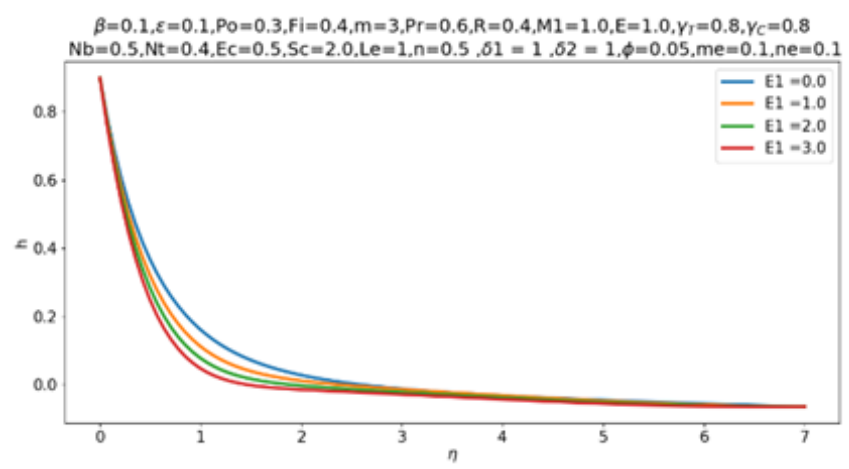

Fig 4. Concentration $[\mathrm{h}(\eta)]$ via Electric parameter (E1)

\section{Execution of radiation parameter (R) on $\theta(\eta)$}

Figure 5 scrutinized that intensifying value of $\mathrm{R}$ present analogous impact and enhance both the temperature and thermal layer thickness. This causes that higher estimation of radiation relates to the rapid vibration of nanoparticles.

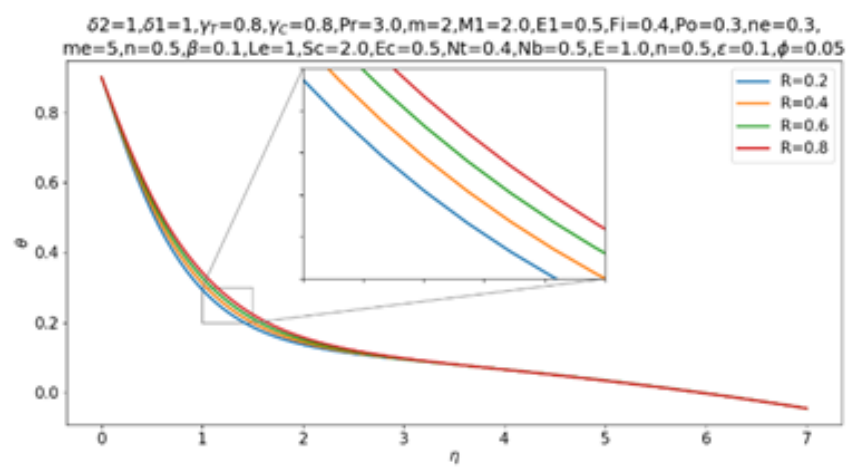

Fig 5. Temperature $[\theta(\eta)]$ via radiation parameter(R)

\section{Execution of SWCNT and MWCNT on $\mathbf{f}^{1}(\eta), \mathbf{g}^{1}(\eta), \theta(\eta)$, and $h(\eta)$}

Figures 6, 7, 8 and 9 Capture the behavior of SWCNT and MWCNT with different base fluids on Momentum, thermal, and concentration boundary layers respectively. The influence of momentum and concentration boundary layers with different base fluids is dominated by MWCNT. The thermal boundary layer is henpecked by SWCNT because of specific heat. 


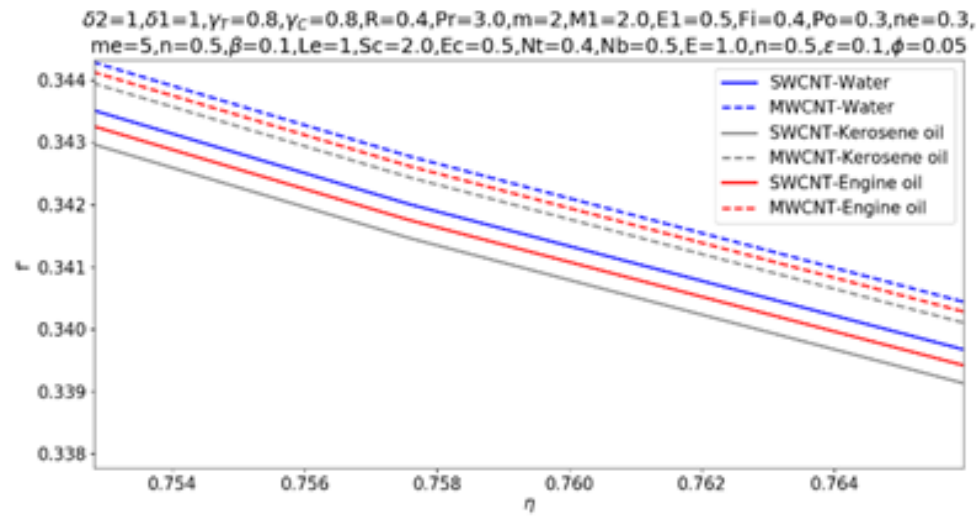

Fig 6. Velocity $\left[\mathrm{f}^{1}(\eta)\right]$ via SWCNT and MWCNT with different base fluids

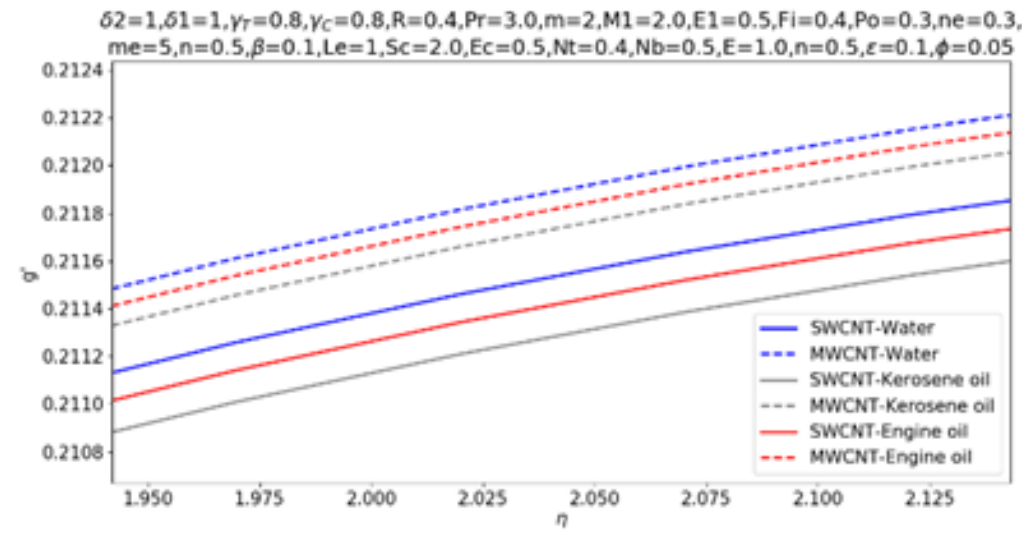

Fig 7. Vertical velocity $\left[g^{1}(\eta)\right]$ via SWCNT and MWCNT with different base fluids

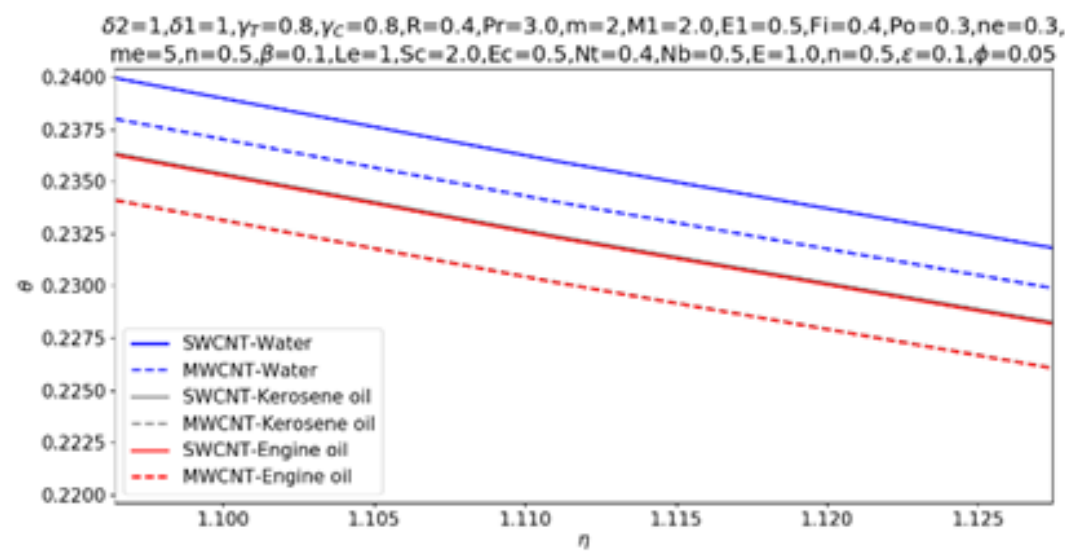

Fig 8. Temperature $[\theta(\eta)]$ via SWCNT and MWCNT with different base fluids 


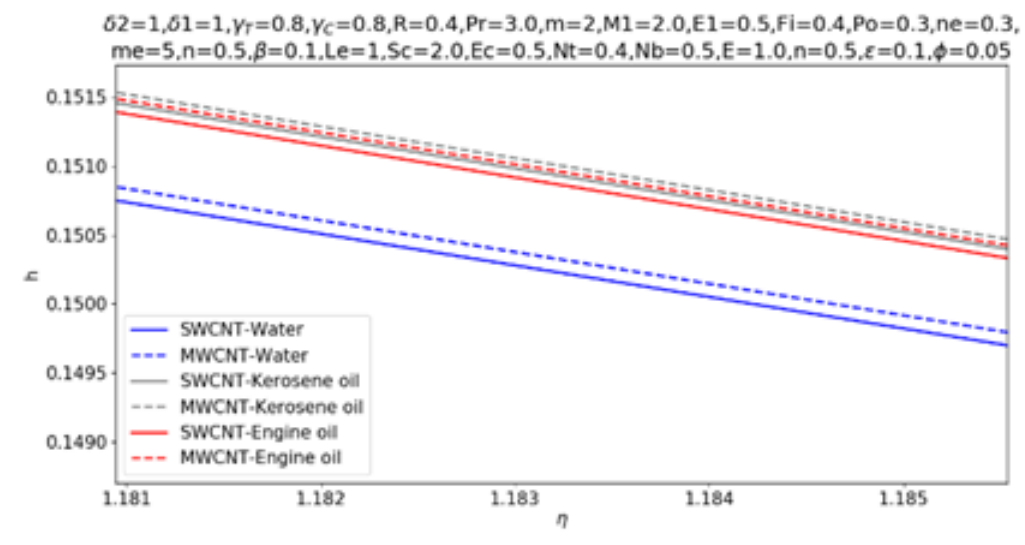

Fig 9. Concentration $[h(\eta)]$ via SWCNT and MWCNT with different base fluids

\section{Tables of local skin friction coefficients, local Nusselt and Sherwood numbers}

Behaviors of magnetic parameter(M1), Electric parameter (E1), power of exponential stretching sheet parameter(m), rotation parameter $(\varepsilon)$, porosity parameter $(\mathrm{Po})$, friction parameter $(\mathrm{Fi})$, nanoparticle volume fraction $(\phi)$ on skin friction coefficient, local Nusselt number, and local Sherwood number are disclosed in Tables 4, 5, 6, 7, 8 and 9. Investigators interpreted that the skin friction coefficient calibrate for excellent estimations of magnetic parameter, and electric parameter and the local skin friction coefficient crumble with growing power of exponential stretching sheet parameter, porosity parameter, friction parameter, and nanoparticle volume fraction. The local Nusselt and Sherwood numbers boots for magnifying magnetic parameter, Electric parameter, power of exponential stretching sheet parameter, and nanoparticle volume fraction and the local Nusselt and Sherwood numbers wane for higher estimations of rotation parameter, porosity parameter, and friction parameter. Influence of various base fluids explored with multi wall carbon nanotube (MWCNT) on local skin friction coefficient are dominated as compared to single wall carbon nanotube (SWCNT), however contradiction appeared in local Nusselt and Sherwood numbers, due to specific heat of nanoparticles.

Table 4. Coefficients of Skin Friction explored with water-based CNT

\begin{tabular}{|c|c|c|c|c|}
\hline & $\left(\operatorname{Re}_{x}\right)^{0.5} C_{f_{x}}$ & & $\left(\operatorname{Re}_{x}\right)^{0.5} C_{f}$ & \\
\hline $\mathrm{m}$ & SWCNT & MWCNT & SWCNT & MWCNT \\
\hline 1 & -0.763489 & -0.729187 & 0.625432 & 0.635101 \\
\hline 2 & -1.224687 & -1.181661 & 0.446345 & 0.455891 \\
\hline 3 & -1.57556 & -1.52556 & 0.327124 & 0.336464 \\
\hline 4 & -1.86877 & -1.812759 & 0.23580 & 0.244967 \\
\hline 5 & -2.12531 & -2.063925 & 0.16076 & 0.169792 \\
\hline$\varepsilon$ & SWCNT & MWCNT & SWCNT & MWCNT \\
\hline 0.0 & -1.271191 & -1.228003 & 0.539242 & 0.550536 \\
\hline 0.2 & -1.182163 & -1.139248 & 0.349305 & 0.356936 \\
\hline 0.3 & -1.143789 & -1.10093 & 0.248363 & 0.253903 \\
\hline 0.4 & -1.110039 & -1.067211 & 0.143628 & 0.146883 \\
\hline 0.5 & -1.081313 & -1.038493 & 0.035255 & 0.036012 \\
\hline M1 & SWCNT & MWCNT & SWCNT & MWCNT \\
\hline 0.0 & -1.6965701 & -1.661854 & -0.246815 & -0.245177 \\
\hline 0.2 & -1.5836861 & -1.546905 & -0.064564 & -0.061061 \\
\hline 0.3 & -1.5338970 & -1.496195 & 0.010431 & 0.014825 \\
\hline 0.4 & -1.4861684 & -1.447600 & 0.080569 & 0.085799 \\
\hline 0.5 & -1.4397164 & -1.400314 & 0.147272 & 0.153316 \\
\hline E1 & SWCNT & MWCNT & SWCNT & MWCNT \\
\hline 0.0 & -1.699203 & -1.664444 & -0.247223 & -0.245606 \\
\hline 0.2 & -1.593936 & -1.557422 & -0.063868 & -0.060351 \\
\hline 0.3 & -1.544436 & -1.50703 & 0.010794 & 0.0151835 \\
\hline
\end{tabular}

Continued on next page 


\begin{tabular}{|c|c|c|c|c|}
\hline \multicolumn{5}{|c|}{ Table 4 continued } \\
\hline 0.4 & -1.496245 & -1.457975 & 0.080603 & 0.0858131 \\
\hline 0.5 & -1.448822 & -1.409697 & 0.147055 & 0.1530623 \\
\hline $\mathrm{Fi}$ & SWCNT & MWCNT & SWCNT & MWCNT \\
\hline 0.0 & -1.102136 & -1.0615124 & 0.457235 & 0.4667986 \\
\hline 0.2 & -1.164633 & -1.1227866 & 0.451751 & 0.4613062 \\
\hline 0.3 & -1.194949 & -1.1525079 & 0.449038 & 0.4585883 \\
\hline 0.4 & -1.224687 & -1.1816615 & 0.446345 & 0.4558919 \\
\hline 0.5 & -1.253877 & -1.2102766 & 0.443676 & 0.4532180 \\
\hline Po & SWCNT & MWCNT & SWCNT & MWCNT \\
\hline 0.1 & -1.101448 & -1.0981814 & 0.490696 & 0.4858830 \\
\hline 0.2 & -1.184205 & -1.1402346 & 0.460712 & 0.4706713 \\
\hline 0.3 & -1.224687 & -1.1816615 & 0.446345 & 0.4558919 \\
\hline 0.4 & -1.264562 & -1.222444 & 0.432384 & 0.4415427 \\
\hline 0.5 & -1.303839 & -1.2625935 & 0.418824 & 0.4276155 \\
\hline$\Phi$ & SWCNT & MWCNT & SWCNT & MWCNT \\
\hline 0.1 & -1.049317 & -1.3334205 & 0.4647983 & 0.4448191 \\
\hline 0.2 & -1.901082 & -1.7158230 & 0.3777966 & 0.4129515 \\
\hline 0.3 & -2.556459 & -2.2570260 & 0.3095976 & 0.3599152 \\
\hline 0.4 & -3.514040 & -3.0773249 & 0.2037653 & 0.2669620 \\
\hline 0.5 & -5.048507 & -4.4423350 & 0.0224635 & 0.2669620 \\
\hline
\end{tabular}

Table 5. Coefficients of Local Nussult and Sherwood numbers explored with water-based CNT

\begin{tabular}{|c|c|c|c|c|}
\hline & $\left(\operatorname{Re}_{x}\right)^{0.5} N u_{x}$ & & $\left(\operatorname{Re}_{x}\right)^{0.5} S h_{x}$ & \\
\hline $\mathrm{m}$ & SWCNT & MWCNT & SWCNT & MWCNT \\
\hline 1 & 0.881598 & 0.869451 & 1.426537 & 1.425611 \\
\hline 2 & 1.274521 & 1.255594 & 1.898613 & 1.896743 \\
\hline 3 & 1.545930 & 1.522809 & 2.262747 & 2.260220 \\
\hline 4 & 1.763793 & 1.737547 & 2.570623 & 2.567568 \\
\hline 5 & 1.950197 & 1.921430 & 2.84245 & 2.838948 \\
\hline$\varepsilon$ & SWCNT & MWCNT & SWCNT & MWCNT \\
\hline 0.0 & 1.268236 & 1.250146 & 1.906029 & 1.904407 \\
\hline 0.2 & 1.276328 & 1.256745 & 1.890306 & 1.888183 \\
\hline 0.3 & 1.273219 & 1.253118 & 1.880995 & 1.878606 \\
\hline 0.4 & 1.265198 & 1.244706 & 1.870577 & 1.867905 \\
\hline 0.5 & 1.2518681 & 1.231095 & 1.858925 & 1.855941 \\
\hline M1 & SWCNT & MWCNT & SWCNT & MWCNT \\
\hline 0.0 & 0.9136202 & 0.907056 & 1.695696 & 1.693349 \\
\hline 0.2 & 1.1029004 & 1.087909 & 1.761876 & 1.759531 \\
\hline 0.3 & 1.1428116 & 1.126658 & 1.785278 & 1.782976 \\
\hline 0.4 & 1.1732553 & 1.156330 & 1.805731 & 1.803474 \\
\hline 0.5 & 1.1972706 & 1.179773 & 1.824191 & 1.822008 \\
\hline E1 & SWCNT & MWCNT & SWCNT & MWCNT \\
\hline 0.0 & 0.9143792 & 0.90787575 & 1.6955819 & 1.69324518 \\
\hline 0.2 & 1.1502403 & 1.13350599 & 1.7528338 & 1.75037047 \\
\hline 0.3 & 1.2017732 & 1.18338601 & 1.7746351 & 1.77217257 \\
\hline 0.4 & 1.2377825 & 1.21838209 & 1.7944560 & 1.79201247 \\
\hline 0.5 & 1.2620529 & 1.24205332 & 1.8131383 & 1.8107688 \\
\hline $\mathrm{Fi}$ & SWCNT & MWCNT & SWCNT & MWCNT \\
\hline 0.0 & 1.312747 & 1.29214719 & 1.906834 & 1.904767 \\
\hline 0.2 & 1.293330 & 1.27358542 & 1.902595 & 1.900628 \\
\hline 0.3 & 1.283853 & 1.26452120 & 1.900574 & 1.898655 \\
\hline 0.4 & 1.274521 & 1.2555948 & 1.898613 & 1.896743 \\
\hline 0.5 & 1.265332 & 1.2468018 & 1.896709 & 1.8948875 \\
\hline Po & SWCNT & MWCNT & SWCNT & MWCNT \\
\hline 0.1 & 1.331488 & 1.29315858 & 1.912361 & 1.90592792 \\
\hline
\end{tabular}




\begin{tabular}{|c|c|c|c|c|}
\hline \multicolumn{5}{|c|}{ Table 5 continued } \\
\hline 0.2 & 1.293544 & 1.27438548 & 1.903081 & 1.90127314 \\
\hline 0.3 & 1.274521 & 1.25559488 & 1.898613 & 1.89674334 \\
\hline 0.4 & 1.255474 & 1.23677044 & 1.894260 & 1.89233832 \\
\hline 0.5 & 1.236412 & 1.21793229 & 1.890033 & 1.88807146 \\
\hline$\Phi$ & SWCNT & MWCNT & SWCNT & MWCNT \\
\hline 0.1 & 0.9196978 & 1.5159484 & 1.8264048 & 1.93621604 \\
\hline 0.2 & 1.8894791 & 1.9030509 & 1.9686279 & 1.97719181 \\
\hline 0.3 & 2.1233675 & 2.1743915 & 1.9840601 & 1.99759366 \\
\hline 0.4 & 2.3066253 & 2.3773794 & 1.9955089 & 2.01101773 \\
\hline 0.5 & 2.5062949 & 2.5588475 & 2.0084366 & 2.02321381 \\
\hline
\end{tabular}

Table 6. Coefficients of Skin Friction explored with Kerosene-based CNT

\begin{tabular}{|c|c|c|c|c|}
\hline & $\left(\operatorname{Re}_{x}\right)^{0.5} C_{f_{x}}$ & & $\left(\mathrm{Re}_{x}\right)^{0.5} C_{f_{y}}$ & \\
\hline $\mathrm{m}$ & SWCNT & MWCNT & SWCNT & MWCNT \\
\hline 1 & -0.78728270 & -0.7443184 & 0.618794252 & 0.6308222 \\
\hline 2 & -1.25455768 & -1.2006357 & 0.439783454 & 0.4516685 \\
\hline 3 & -1.61029388 & -1.5476063 & 0.320689151 & 0.3323352 \\
\hline 4 & -1.90769964 & -1.8374533 & 0.22947459 & 0.2409181 \\
\hline 5 & -2.16799310 & -2.0909877 & 0.154521663 & 0.1658075 \\
\hline$\varepsilon$ & SWCNT & MWCNT & SWCNT & MWCNT \\
\hline 0.0 & -1.30117773 & -1.2470478 & 0.5315067 & 0.5455338 \\
\hline 0.2 & -1.21194929 & -1.1581743 & 0.3440288 & 0.3535671 \\
\hline 0.3 & -1.17352765 & -1.1198359 & 0.2444878 & 0.2514672 \\
\hline 0.4 & -1.13975052 & -1.0861030 & 0.1412802 & 0.1454674 \\
\hline 0.5 & -1.11101399 & -1.0573839 & 0.0346131 & 0.0357135 \\
\hline M1 & SWCNT & MWCNT & SWCNT & MWCNT \\
\hline 0.0 & -1.7208377 & -1.6771385 & -0.247993 & -0.2458919 \\
\hline 0.2 & -1.6093339 & -1.5631009 & -0.067017 & -0.0626021 \\
\hline 0.3 & -1.5601657 & -1.5128010 & 0.007372 & 0.0128899 \\
\hline 0.4 & -1.5126781 & -1.4645914 & 0.077107 & 0.0834923 \\
\hline 0.5 & -1.4671338 & -1.4176768 & 0.143098 & 0.1506492 \\
\hline E1 & SWCNT & MWCNT & SWCNT & MWCNT \\
\hline 0.0 & -1.72348847 & -1.6797401 & -0.248387 & -0.2463107 \\
\hline 0.2 & -1.61940465 & -1.5734994 & -0.066330 & -0.0618979 \\
\hline 0.3 & -1.57050528 & -1.5235053 & 0.007737 & 0.0132498 \\
\hline 0.4 & -1.52290030 & -1.4748334 & 0.076989 & 0.0835152 \\
\hline 0.5 & -1.47605425 & -1.4269363 & 0.142899 & 0.1504102 \\
\hline $\mathrm{Fi}$ & SWCNT & MWCNT & SWCNT & MWCNT \\
\hline 0.0 & -1.1303323 & -1.0794290 & 0.4506578 & 0.4625680 \\
\hline 0.2 & -1.1936816 & -1.1412416 & 0.4451820 & 0.4570793 \\
\hline 0.3 & -1.2244121 & -1.1712247 & 0.4424721 & 0.4543632 \\
\hline 0.4 & -1.2545576 & -1.2006357 & 0.4397834 & 0.4516685 \\
\hline 0.5 & -1.2841480 & -1.2295038 & 0.4371175 & 0.4489967 \\
\hline Po & SWCNT & MWCNT & SWCNT & MWCNT \\
\hline 0.1 & -1.17430154 & -1.1180172 & 0.4683628 & 0.4812823 \\
\hline 0.2 & -1.21470827 & -1.1596305 & 0.4538733 & 0.4662640 \\
\hline 0.3 & -1.25455768 & -1.2006357 & 0.4397834 & 0.4516685 \\
\hline 0.4 & -1.29382331 & -1.2410140 & 0.4260831 & 0.4374925 \\
\hline 0.5 & -1.33251439 & -1.2807739 & 0.4127686 & 0.4237287 \\
\hline$\Phi$ & SWCNT & MWCNT & SWCNT & MWCNT \\
\hline 0.1 & -1.48106727 & -1.372421 & 0.413824727 & 0.4365221 \\
\hline 0.2 & -2.02385201 & -1.799084 & 0.355116802 & 0.3969613 \\
\hline 0.3 & -2.75084994 & -2.392287 & 0.27800306 & 0.3369254 \\
\hline 0.4 & -3.79505556 & -3.275272 & 0.16423471 & 0.2380382 \\
\hline 0.5 & -5.43889221 & -4.717023 & -0.02419145 & 0.0624988 \\
\hline
\end{tabular}


Table 7. Coefficients of Local Nussult number and Sherwood number explored with Kerosene-based CNT

\begin{tabular}{|c|c|c|c|c|}
\hline & $\left(\operatorname{Re}_{x}\right)^{0.5} N u_{x}$ & & $\left(\operatorname{Re}_{x}\right)^{0.5} S h_{x}$ & \\
\hline $\mathrm{m}$ & SWCNT & MWCNT & SWCNT & MWCNT \\
\hline 1 & 0.838838344 & 0.82985086 & 1.4151419 & 1.41365674 \\
\hline 2 & 1.213268473 & 1.19820199 & 1.8820387 & 1.87932240 \\
\hline 3 & 1.47244527 & 1.45377823 & 2.2423974 & 2.23880775 \\
\hline 4 & 1.68077515 & 1.65950504 & 2.5471413 & 2.54284284 \\
\hline 5 & 1.85920227 & 1.83588313 & 2.8162258 & 2.81132052 \\
\hline$\varepsilon$ & SWCNT & MWCNT & SWCNT & MWCNT \\
\hline 0.0 & 1.2066017 & 1.19254704 & 1.8895487 & 1.88711932 \\
\hline 0.2 & 1.2156722 & 1.19979434 & 1.8736804 & 1.87066823 \\
\hline 0.3 & 1.2134269 & 1.19688527 & 1.8643814 & 1.86105674 \\
\hline 0.4 & 1.2065505 & 1.18948258 & 1.8540344 & 1.85037131 \\
\hline 0.5 & 1.1947667 & 1.17720988 & 1.8425280 & 1.83848151 \\
\hline M1 & SWCNT & MWCNT & SWCNT & MWCNT \\
\hline 0.0 & 0.8789959 & 0.8762448 & 1.6800026 & 1.67671568 \\
\hline 0.2 & 1.0535758 & 1.0419437 & 1.7465854 & 1.74327940 \\
\hline 0.3 & 1.0904923 & 1.0777651 & 1.7698048 & 1.76655332 \\
\hline 0.4 & 1.1181178 & 1.1053161 & 1.7902317 & 1.78687889 \\
\hline 0.5 & 1.1410841 & 1.1271501 & 1.8083639 & 1.80525805 \\
\hline E1 & SWCNT & MWCNT & SWCNT & MWCNT \\
\hline 0.0 & 0.87958907 & 0.87690724 & 1.6799112 & 1.67664138 \\
\hline 0.2 & 1.098745825 & 1.08510138 & 1.7371641 & 1.73374883 \\
\hline 0.3 & 1.146604947 & 1.13132109 & 1.7586978 & 1.75529094 \\
\hline 0.4 & 1.180061792 & 1.16381597 & 1.7782936 & 1.77491261 \\
\hline 0.5 & 1.202585929 & 1.18581340 & 1.7967989 & 1.79350819 \\
\hline $\mathrm{Fi}$ & SWCNT & MWCNT & SWCNT & MWCNT \\
\hline 0.0 & 1.25027263 & 1.2333168 & 1.8897660 & 1.88684883 \\
\hline 0.2 & 1.23147766 & 1.2154869 & 1.8857754 & 1.88296004 \\
\hline 0.3 & 1.22230241 & 1.2067787 & 1.8838771 & 1.88111161 \\
\hline 0.4 & 1.21326847 & 1.1982019 & 1.8820387 & 1.87932240 \\
\hline 0.5 & 1.20437101 & 1.1897524 & 1.8802569 & 1.87758921 \\
\hline Po & SWCNT & MWCNT & SWCNT & MWCNT \\
\hline 0.1 & 1.24899191 & 1.23350801 & 1.8905160 & 1.88797419 \\
\hline 0.2 & 1.23114124 & 1.21586512 & 1.8862191 & 1.88358656 \\
\hline 0.3 & 1.21326847 & 1.19820199 & 1.8820387 & 1.87932240 \\
\hline 0.4 & 1.19537341 & 1.18050540 & 1.8779686 & 1.87518054 \\
\hline 0.5 & 1.17746296 & 1.16279403 & 1.8740173 & 1.87116903 \\
\hline$\Phi$ & SWCNT & MWCNT & SWCNT & MWCNT \\
\hline 0.1 & 1.44120937 & 1.4311677 & 1.9122720 & 1.91179294 \\
\hline 0.2 & 1.78364744 & 1.81440513 & 1.9423220 & 1.94872670 \\
\hline 0.3 & 2.03801353 & 2.12448323 & 1.9584476 & 1.96998616 \\
\hline 0.4 & 2.24434980 & 2.38133492 & 1.9727971 & 1.98660227 \\
\hline 0.5 & 2.43371574 & 2.59387775 & 1.9902222 & 2.00364969 \\
\hline
\end{tabular}

Table 8. Coefficients of Skin Friction explored with engine oil-based CNT

\begin{tabular}{lllll}
\hline & $\left(\operatorname{Re}_{x}\right)^{0.5} C_{f_{x}}$ & & $\left(\operatorname{Re}_{x}\right)^{0.5} C_{f_{y}}$ \\
\hline $\mathrm{m}$ & SWCNT & MWCNT & SWCNT & MWCNT \\
\hline 1 & -1.4076195 & -0.7443184 & 0.42762256 & 0.6308222 \\
2 & -1.6572581 & -1.2006357 & 0.40370852 & 0.4516685 \\
3 & -1.8782380 & -1.5476063 & 0.38235177 & 0.3323352 \\
4 & -2.0783363 & -1.8374533 & 0.36319958 & 0.2409181 \\
5 & -2.2624266 & -2.0909877 & 0.34590040 & 0.1658075 \\
\hline$\varepsilon$ & SWCNT & MWCNT & SWCNT & MWCNT \\
\hline 0.0 & -1.28529361 & -1.2369439 & 0.5355939 & 0.5481834 \\
\hline
\end{tabular}

Continued on next page 


\begin{tabular}{|c|c|c|c|c|}
\hline \multicolumn{5}{|c|}{ Table 8 continued } \\
\hline 0.2 & -1.19617209 & -1.1481338 & 0.3468219 & 0.3553536 \\
\hline 0.3 & -1.15777691 & -1.1098087 & 0.2465438 & 0.2527610 \\
\hline 0.4 & -1.12401526 & -1.0760812 & 0.1425328 & 0.1462226 \\
\hline 0.5 & -1.09528198 & -1.0473633 & 0.0349485 & 0.0358790 \\
\hline M1 & SWCNT & MWCNT & SWCNT & MWCNT \\
\hline 0.0 & -1.70797226 & -1.6690275 & -0.247366 & -0.2455113 \\
\hline 0.2 & -1.59573715 & -1.5545037 & -0.065716 & -0.0617840 \\
\hline 0.3 & -1.54624188 & -1.5039871 & 0.0089939 & 0.0139173 \\
\hline 0.4 & -1.49843913 & -1.4555736 & 0.0790343 & 0.0847163 \\
\hline 0.5 & -1.45260471 & -1.4084628 & 0.1453097 & 0.1520634 \\
\hline E1 & SWCNT & MWCNT & SWCNT & MWCNT \\
\hline 0.0 & -1.71060950 & -1.6716188 & -0.247766 & -0.2459352 \\
\hline 0.2 & -1.60590240 & -1.5649650 & -0.065024 & -0.0610765 \\
\hline 0.3 & -1.55668643 & -1.5147617 & 0.009357 & 0.0142761 \\
\hline 0.4 & -1.50877261 & -1.4658860 & 0.078903 & 0.0847343 \\
\hline 0.5 & -1.46162259 & -1.4177878 & 0.1450991 & 0.1518167 \\
\hline $\mathrm{Fi}$ & SWCNT & MWCNT & SWCNT & MWCNT \\
\hline 0.0 & -1.11539820 & -1.0699240 & 0.454135 & 0.4648099 \\
\hline 0.2 & -1.17829564 & -1.1314509 & 0.448655 & 0.4593192 \\
\hline 0.3 & -1.20880637 & -1.1612949 & 0.445943 & 0.4566021 \\
\hline 0.4 & -1.23873587 & -1.1905693 & 0.443252 & 0.4539065 \\
\hline 0.5 & -1.26811386 & -1.2193031 & 0.440585 & 0.4512337 \\
\hline Po & SWCNT & MWCNT & SWCNT & MWCNT \\
\hline 0.1 & -1.15779979 & -1.1074957 & 0.4721304 & 0.4837195 \\
\hline 0.2 & -1.19855372 & -1.1493414 & 0.4574886 & 0.4685991 \\
\hline 0.3 & -1.23873587 & -1.1905693 & 0.4432529 & 0.4539065 \\
\hline 0.4 & -1.27832218 & -1.2311616 & 0.4294155 & 0.4396389 \\
\hline 0.5 & -1.31732151 & -1.2711269 & 0.4159714 & 0.4257888 \\
\hline$\phi$ & SWCNT & MWCNT & SWCNT & MWCNT \\
\hline 0.1 & -1.44950364 & -1.3517839 & 0.4203567675 & 0.4409025 \\
\hline 0.2 & -1.95938268 & -1.7552095 & 0.3669605953 & 0.4053546 \\
\hline 0.3 & -2.64907930 & -2.3212162 & 0.2944445106 & 0.3489499 \\
\hline 0.4 & -3.64813906 & -3.1714246 & 0.1847969512 & 0.2531525 \\
\hline 0.5 & -5.23476384 & -4.5728929 & 0.0001387517 & 0.0800308 \\
\hline
\end{tabular}

Table 9. Coefficients of Local Nussult number and Sherwood number explored with engine oil based CNT

\begin{tabular}{lllll}
\hline & $\left(\mathrm{Re}_{x}\right)^{0.5} N u_{x}$ & & $\left(\mathrm{Re}_{x}\right)^{0.5} S h_{x}$ \\
\hline $\mathrm{m}$ & SWCNT & MWCNT & SWCNT & MWCNT \\
\hline 1 & 1.16679601 & 0.82985086 & 1.8735376 & 1.41365674 \\
2 & 1.09016705 & 1.19820199 & 1.8601454 & 1.87932240 \\
3 & 1.02192905 & 1.45377823 & 1.8495870 & 2.23880775 \\
4 & 0.96021831 & 1.65950501 & 1.8410352 & 2.54284284 \\
5 & 0.90371873 & 1.83588313 & 1.8339723 & MWCNT \\
\hline$\varepsilon$ & SWCNT & MWCNT & SWCNT & 1.8881878 \\
0.0 & 1.2115332 & 1.19496544 & 1.8910634 & 1.8716645 \\
0.2 & 1.2200689 & 1.20188217 & 1.8750855 & 1.8620066 \\
0.3 & 1.2175664 & 1.19881388 & 1.8657164 & 1.8512660 \\
0.4 & 1.2104294 & 1.19124960 & 1.8552864 & 1.8393098 \\
0.5 & 1.1982907 & 1.17880587 & 1.8436710 & MWCNT \\
\hline M1 & SWCNT & MWCNT & SWCNT & 1.67758681 \\
\hline 0.0 & 0.88311093 & 0.87827153 & 1.6812307 & 1.74411655 \\
0.2 & 1.05749993 & 1.04381688 & 1.7477606 & 1.76741415 \\
0.3 & 1.09453921 & 1.07970267 & 1.7710148 & 1.78776390 \\
0.4 & 1.12227104 & 1.10731410 & 1.7914838 & 1.80617125 \\
0.5 & 1.14534255 & 1.12919763 & 1.8096495 & \\
\hline
\end{tabular}

Continued on next page 


\begin{tabular}{|c|c|c|c|c|}
\hline \multicolumn{5}{|c|}{ Table 9 continued } \\
\hline E1 & SWCNT & MWCNT & SWCNT & MWCNT \\
\hline 0.0 & 0.8837367 & 0.87895566 & 1.6811416 & 1.67751344 \\
\hline 0.2 & 1.1024795 & 1.08686166 & 1.7383636 & 1.73459986 \\
\hline 0.3 & 1.1504187 & 1.13312086 & 1.7599330 & 1.75616572 \\
\hline 0.4 & 1.1839737 & 1.16566653 & 1.7795624 & 1.77581075 \\
\hline 0.5 & 1.2065971 & 1.18771558 & 1.7981092 & 1.79443530 \\
\hline $\mathrm{Fi}$ & SWCNT & MWCNT & SWCNT & MWCNT \\
\hline 0.0 & 1.25465663 & 1.2353866 & 1.8912477 & 1.88789578 \\
\hline 0.2 & 1.23600030 & 1.21764751 & 1.8872482 & 1.88400100 \\
\hline 0.3 & 1.22689189 & 1.20898303 & 1.8853453 & 1.88214952 \\
\hline 0.4 & 1.21792324 & 1.20044912 & 1.8835023 & 1.88035721 \\
\hline 0.5 & 1.20908960 & 1.19204140 & 1.8817159 & 1.87862090 \\
\hline Po & SWCNT & MWCNT & SWCNT & MWCNT \\
\hline 0.1 & 1.25384014 & 1.23587080 & 1.8920906 & 1.88908341 \\
\hline 0.2 & 1.23589200 & 1.21817013 & 1.8877372 & 1.88465776 \\
\hline 0.3 & 1.21792324 & 1.20044912 & 1.8835023 & 1.88035721 \\
\hline 0.4 & 1.19992910 & 1.18269312 & 1.8793809 & 1.87618108 \\
\hline 0.5 & 1.18191964 & 1.16492279 & 1.8753815 & 1.87213790 \\
\hline$\phi$ & SWCNT & MWCNT & SWCNT & MWCNT \\
\hline 0.1 & 1.451888367 & 1.43652160 & 1.9150944 & 1.91381368 \\
\hline 0.2 & 1.807616662 & 1.82721072 & 1.9471442 & 1.95227920 \\
\hline 0.3 & 2.074458380 & 2.14474563 & 1.9642875 & 1.97435416 \\
\hline 0.4 & 2.290496077 & 2.40732995 & 1.9787338 & 1.99106822 \\
\hline 0.5 & 2.486432966 & 2.62236855 & 1.9954937 & 2.00759103 \\
\hline
\end{tabular}

\section{Conclusion}

A numerical spatial simulation of the Darcy -Forchhiemer flow explored with SWCNT and MWCNT on stretching in the presence of Joule heating, hall current with ion slip, and activation energy are carried out. The flow generating equations are converted into a system of coupled nonlinear ODEs and are solved numerically with python coding. The interesting conclusions of the present work are presented as follows:

- Electric field parameter has an important enhancing influence on velocity, temperature, and opposite nature on concentration. Investigators observed that the intermolecular force of CNT is the cause to reduce the concentration and increase the velocity and temperature.

- Velocity, concentration enhances and temperature decreases with increasing porosity. Researchers exploit the fact that absorption is the major cause of nanofluid explored with CNT.

- Investigators determined that temperature and concentration are positively correlated to inertial resistance, Velocity and inertial resistance are negatively correlated due to friction of CNT in a nanofluid.

- Investigators noticed that due to thermal conductivity and specific heat of Water, water-based carbon nanotube (SWCNT and MWCNT) profiles are enhanced when compared to the remaining base fluids except at concentration profile, because of the thermophoretic parameter of CNT.

This investigation is limited to study the deformation of nanofluids with carbon nanotubes with different base fluids using boundary value problems through python coding. Nowadays python code plays an important role to solve the nonlinear coupled ordinary differential equations to estimate the deformation of nanofluids even in the case of hybrid carbon nanotubes also.

\section{Conflict of Interest}

Investigators confirm that there is no conflict of interest to declare for this publication. 


\section{Nomenclature}

\begin{tabular}{|c|c|c|c|}
\hline $\mathrm{B}_{0}$ & Constant magnetic field intensity & $\mathrm{m}$ & Power of the exponential stretching sheet. \\
\hline$c_{f}$ & Inertial resistance & M1 & Electric parameter \\
\hline$c_{p}$ & Specific heat. & $\mathrm{N}$ & Fitted rate coefficient \\
\hline $\mathrm{C}_{w}$ & Wall concentration & ne & Hall current parameter \\
\hline$C_{\infty}$ & Concentration outside the boundary layer & ni & Ion-slip \\
\hline $\mathrm{D}_{B}$ & Brownion diffusion coefficient. & $\mathrm{Nb}$ & Brownian motion parameter \\
\hline $\mathrm{D}_{T}$ & Thermal diffusion coefficient. & $\mathrm{Nt}$ & Thermophoresis parameter \\
\hline $\mathrm{E}$ & Activation energy parameter & $\mathrm{P}$ & Velocity components in $\mathrm{x}$ direction \\
\hline $\mathrm{E}_{0}$ & Constant electric field intensity & $\mathrm{P}$ & Reference parameter \\
\hline E1 & Electric parameter & Po & Porosity parameter \\
\hline$E^{*}$ & Activation energy & $\operatorname{Pr}$ & Prandtl number \\
\hline $\mathrm{F}_{i}$ & Inertial resistance parameter & Q & Velocity components in y direction \\
\hline $\mathrm{K}$ & Boltzman constant & $\mathrm{r}$ & Velocity components in $\mathrm{z}$ direction \\
\hline $\mathrm{k}_{r}$ & Reaction rate parameter. & $\mathrm{R}$ & Radiation parameter \\
\hline $\mathrm{K}_{b f}$ & Thermal conductivity of basefluid & Sc & Schmidt number \\
\hline $\mathrm{K}_{C N T}$ & Thermal conductivity of CNT & $\mathrm{T}_{w}$ & Wall temperature \\
\hline $\mathrm{K}_{n f}$ & Thermal conductivity of nanofluid & $\mathrm{T}_{\infty}$ & Temperature of the fluid outside the boundary layer \\
\hline $\mathrm{K}^{*}$ & Permeability of porous media & $\mathrm{x}, \mathrm{y}, \mathrm{z}$ & Cartesian coordinates \\
\hline $\mathrm{L}$ & Reference length. & $\beta$ & Ratio of stretching rates parameter \\
\hline Le & Lewies number & $\varepsilon$ & Rotation parameter \\
\hline \multirow[t]{2}{*}{ me } & Hall parameter & & \\
\hline & & $\gamma_{C}$ & Concentration Biot numbers \\
\hline$\gamma_{T}$ & Thermal Biot numbers & $\eta$ & Similarity variable \\
\hline$\delta 1$ & Temperature difference parameter & $\sigma_{n f}$ & Electrical conductivity of nanofluid \\
\hline$\delta 2$ & Reaction rate parameter & $\sigma_{b f}$ & Electrical conductivity of basefluid \\
\hline$\rho_{b f}$ & Density of basefluid & $\sigma_{C N T}$ & Electrical conductivity of CNT \\
\hline$\rho_{C N T}$ & Density of CNT & $\mu_{n f}$ & Coefficient of viscosity of nanofluid \\
\hline$\phi$ & Nanoparticle volume fraction & $\mu_{b f}$ & Coefficient of viscosity of basefluid \\
\hline$\theta$ & Dimensionless fluid temperature & $v_{n f}$ & Coefficient of kinematics viscosity of nanofluid \\
\hline
\end{tabular}

\section{References}

1) Hayat T, Shafiq A, Alsaedi A. Effect of Joule Heating and Thermal Radiation in Flow of Third Grade Fluid over Radiative Surface. PLoS ONE. 2014;9(1):e83153. Available from: https://dx.doi.org/10.1371/journal.pone.0083153.

2) Hayat T, Muhammad T, Shehzad SA, Alsaedi A. Similarity solution to three dimensional boundary layer flow of second grade nanofluid past a stretching surface with thermal radiation and heat source/sink. AIP Advances. 2015;5(1). Available from: https://dx.doi.org/10.1063/1.4905780.

3) Sreenivasulu P, Reddy NB, and. Lie Group Analysis for Boundary Layer Flow of Nanofluids near the Stagnation-Point over a Permeable Stretching Surface Embedded in a Porous Medium in the Presence of Radiation and Heat Generation/Absorption. Journal of Applied Fluid Mechanics. 2015;8(3):549-558. Available from: https://dx.doi.org/10.18869/acadpub.jafm.67.222.19373.

4) Khan U, Ahmed N, Mohyud-Din ST. Influence of viscous dissipation and Joule heating on MHD bio-convection flow over a porous wedge in the presence of nanoparticles and gyrotactic microorganisms. SpringerPlus. 2016;5(1). Available from: https://dx.doi.org/10.1186/s40064-016-3718-8.

5) Afshar H, Shams M, Naeinian MM, Ahmadi G. Effect of Dispersed Nanoparticles on Thermophysical Properties of Nanofluid and Heat Transfer Coeficients. Journal of Applied Fluid Mechanics. 2016;9:165-171. Available from: 10.36884/jafm.9.SI1.25817.

6) Sreenivasulu P, Poornima T, Reddy NB. Influence of Joule Heating and Non-Linear Radiation on MHD 3D Dissipating Flow of Casson Nanofluid past a Non-Linear Stretching Sheet. Nonlinear Engineering. 2019;8(1):661-672. Available from: https://dx.doi.org/10.1515/nleng-2017-0143.

7) Shahzad F, Sagheer M, Hussain S. Numerical simulation of magnetohydrodynamic Jeffrey nanofluid flow and heat transfer over a stretching sheet considering Joule heating and viscous dissipation. AIP Advances. 2018;8(6). Available from: https://dx.doi.org/10.1063/1.5031447.

8) Reddy JVR, Sugunamma V, Sandeep N. Simultaneous impacts of Joule heating and variable heat source/sink on MHD 3D flow of Carreau-nanoliquids with temperature dependent viscosity. Nonlinear Engineering. 2019;8(1):356-367. Available from: https://dx.doi.org/10.1515/nleng-2017-0132.

9) Gholinia M, Gholinia S, Hosseinzadeh K, Ganji DD. Investigation on ethylene glycol Nano fluid flow over a vertical permeable circular cylinder under effect of magnetic field. Results in Physics. 2018;9:1525-1533. Available from: https://dx.doi.org/10.1016/j.rinp.2018.04.070.

10) Singh J, Gupta M, Kumar R, Kumar H. Heat Transfer using Nanofluid. International Journal of Engineering and Advanced Technology (IJEAT). 2019;9(2):3205-3211. Available from: 10.35940/ijeat.B9230.129219.

11) Srinivasacharya D, Jagadeeshwar P. Effect of Joule heating on the flow over an exponentially stretching sheet with convective thermal condition. Mathematical Sciences. 2019;13(3):201-211. Available from: https://dx.doi.org/10.1007/s40096-019-0290-8. 
12) Tlili I, Nabwey HA, Ashwinkumar GP, Sandeep N. 3-D magnetohydrodynamic AA7072-AA7075/methanol hybrid nanofluid flow above an uneven thickness surface with slip effect. Scientific Reports. 2020;10(1). Available from: https://dx.doi.org/10.1038/s41598-020-61215-8.

13) Alzahrani A. Importance of Darcy-Forchheimer porous medium in 3D convective flow of carbon nanotubes. Physics Letters A. 2018;382(40):2938-2943. Available from: https://doi.org/10.1016/j.physleta.2018.06.030.

14) Majeed A, Zeeshan A, Noori FM. Numerical study of Darcy-Forchheimer model with activation energy subject to chemically reactive species and momentum slip of order two. AIP Advances. 2019;9(4). Available from: https://dx.doi.org/10.1063/1.5095546.

15) Muhammad T, Alsaedi A, Hayat T, Shehzad SA. A revised model for Darcy-Forchheimer three-dimensional flow of nanofluid subject to convective boundary condition. Results in Physics. 2017;7:2791-2797. Available from: https://dx.doi.org/10.1016/j.rinp.2017.07.052.

16) Ganesh NV, Hakeem AKA, Ganga B. Darcy-Forchheimer flow of hydromagnetic nanofluid over a stretching/shrinking sheet in a thermally stratified porous medium with second order slip, viscous and Ohmic dissipations effects. Ain Shams Engineering Journal. 2018;9(4):939-951. Available from: https://dx.doi.org/10.1016/j.asej.2016.04.019.

17) Hosseinzadeh K, Roghani SO, Mogharrebi AR, Asadi A, Waqas M, Ganji DD. Investigation of cross-fluid flow containing motile gyrotactic microorganisms and nanoparticles over a three-dimensional cylinder. Alexandria Engineering Journal. 2020;59(5):3297-3307. Available from: https://dx.doi.org/10.1016/ j.aej.2020.04.037.

18) Hosseinzadeh K, Mardani MR, Salehi S, Paikar M, Waqas M, Ganji DD. Entropy generation of three-dimensional Bödewadt flow of water and hexanol base fluid suspended by Fe3O4 and MoS2 hybrid nanoparticles. Journal of Physics. 2021;95(57). Available from: https://dx.doi.org/10.1007/s12043-02002075-9. 